\title{
Case Report: Development of Miliary Pulmonary Tuberculosis in a Patient with Peritoneal Tuberculosis after COVID-19 Upper Respiratory Tract Infection
}

\author{
Moustafa M. Elziny, ${ }^{1 \star}$ Ahmed Ghazy, ${ }^{2}$ Khaled A. Elfert, ${ }^{3}$ and Mohamed Aboukamar ${ }^{4}$ \\ ${ }^{1}$ Department of Family Medicine, Hamad Medical Corporation, Doha, Qatar; ${ }^{2}$ Department of Bariatric Medicine, Hamad Medical Corporation, \\ Doha, Qatar; ${ }^{3}$ Department of Gastroenterology, Hamad Medical Corporation, Doha, Qatar; ${ }^{4}$ Department of Infectious Disease, \\ Hamad Medical Corporation, Doha, Qatar
}

\begin{abstract}
We report a 29-year-old patient who presented with coronavirus disease 2019 (COVID-19) upper respiratory tract infection in addition to clinical, laboratory, and radiological findings highly suggestive of peritoneal tuberculosis (TB) without pulmonary involvement. Two weeks after the resolution of COVID-19 infection, he presented with shortness of breath and oxygen desaturation requiring intubation and admission to the intensive care unit. The workup confirmed miliary pulmonary TB. The patient subsequently improved on antitubercular treatment. We discuss the possible contribution of COVID-19 infection to the rapid progression of TB infection to involve the lung in a miliary pattern, and how the coexistence of the two diseases might have led to a worse outcome.
\end{abstract}

\section{INTRODUCTION}

Tuberculosis (TB) is a disease that roughly affects 10 million people each year. Furthermore, recent estimates suggest that $23 \%$ of the world's population is infected with Mycobacterium tuberculosis. ${ }^{1,2}$ It is transmitted by inhalation of aerosol droplets that contain the organism. It is characterized by the development of necrotizing granulomas that mainly affect the lungs and involve other extrapulmonary sites as well. ${ }^{3}$ Miliary TB occurs as a result of the hematogenous spread of M. tuberculosis. The term miliary refers to the small white nodules that resemble millet seeds. Although miliary TB presentation is more commonly subacute or chronic, it can have acute and even fulminant presentation. The presentation of miliary TB as acute respiratory distress syndrome (ARDS) has been described in two case series. ${ }^{4,5}$

Coronavirus disease 2019 (COVID-19) is a respiratory disease caused by severe acute respiratory syndrome coronavirus 2 (SARS-CoV-2), which is a novel Betacoronavirus. COVID-19 is the disease responsible for the development of the COVID-19 pandemic. The first case was diagnosed in Qatar on February 28, 2020. Afterward, 5,685 cases were diagnosed between February 28 and April 18, 2020. ${ }^{6}$ The common symptoms of COVID-19 infection are fever, cough, malaise, myalgia, and sore throat. Some patients develop gastrointestinal symptoms such as nausea, vomiting, and diarrhea. Most of the patients with COVID-19 have mild to moderate illness, and those with mild disease usually require supportive management only. However, some patients initially present with mild symptoms and later develop more severe illness. ${ }^{7}$

Many studies linked TB reactivation to various viral infections. In 1908, Pirquet $^{8}$ was the first to report a relationship between postmeasles infection and TB reactivation. Also, untreated HIV infection was associated with an increased likelihood of latent TB infection progression to active disease. ${ }^{9}$ Here, we describe a patient who was diagnosed with the TB and COVID-19 infections concurrently. Also, we discuss the impact of COVID-19 on the clinical course of TB.

*Address correspondence to Moustafa M. Elziny, Department of Family Medicine, Hamad Medical Corporation, PO Box 3050, Doha, Qatar. E-mail: drmostafaelzeiny@gmail.com

\section{CASE PRESENTATION}

We report a case of a 29-year-old Nepali male patient, not known to have any chronic illness, who presented on April 13,2020 with a 3-day history of fever and dry cough, and 7 days of abdominal distention. He had no history of shortness of breath, recent weight loss, or night sweats. Also, no history of recent travel, exposure to COVID-19, or TB contacts were reported. There was no history of tobacco or alcohol use. Initially, the patient was vitally stable, maintaining oxygen saturation on room air, and chest examination was unremarkable. Abdominal examination showed a distended, tense abdomen with shifting dullness; he had no tenderness, rebound tenderness, or palpable lymph nodes.

Laboratory investigations showed a white blood cell count of 3,000 cells $/ \mathrm{mm}^{3}$ (70\% neutrophils, $10 \%$ lymphocytes, $17 \%$ monocytes, $2.4 \%$ eosinophils), a C-reactive protein level of $252 \mathrm{mg} / \mathrm{dL}$, and a ferritin level of $746 \mathrm{ug} / \mathrm{L}$. His renal and liver function parameters were within the normal range. An interferon- $\gamma$ release assay was indeterminate. HIV serology was negative. A computed tomographic scan of the abdomen with contrast showed moderate ascites, peritoneal thickening, omental caking, and mesenteric adenopathy, suggesting tuberculous peritonitis or pseudomyxoma peritonei (Figure 1). Chest X-ray on admission was unremarkable (Figure 2).

Peritoneal fluid tapping showed a low serum-ascites albumin gradient $(2.7 \mathrm{~g} / \mathrm{L})$ and a high protein level $(54.1 \mathrm{~g} / \mathrm{L})$, with a lymphocytic predominance (65\%) (Table 1). Peritoneal acidfast bacilli (AFB) smear, culture, and polymerase chain reaction (PCR) were negative. Ascitic fluid bacterial culture was negative. Cytology from the peritoneal fluid was negative for malignancy.

After admission, a SARS-CoV-2 qualitative reverse transcriptase (RT)-PCR tested positive from the nasopharyngeal and throat swabs, so the patient was isolated and received 5 days of oral azithromycin and hydroxychloroquine (per the protocol of the Communicable Disease Center in Qatar). The patient was scheduled for a peritoneal biopsy after the resolution of the COVID-19 infection. During the hospital course, the patient was vitally stable, with no worsening symptoms, and the chest X-ray was repeated and was normal. Repeated 


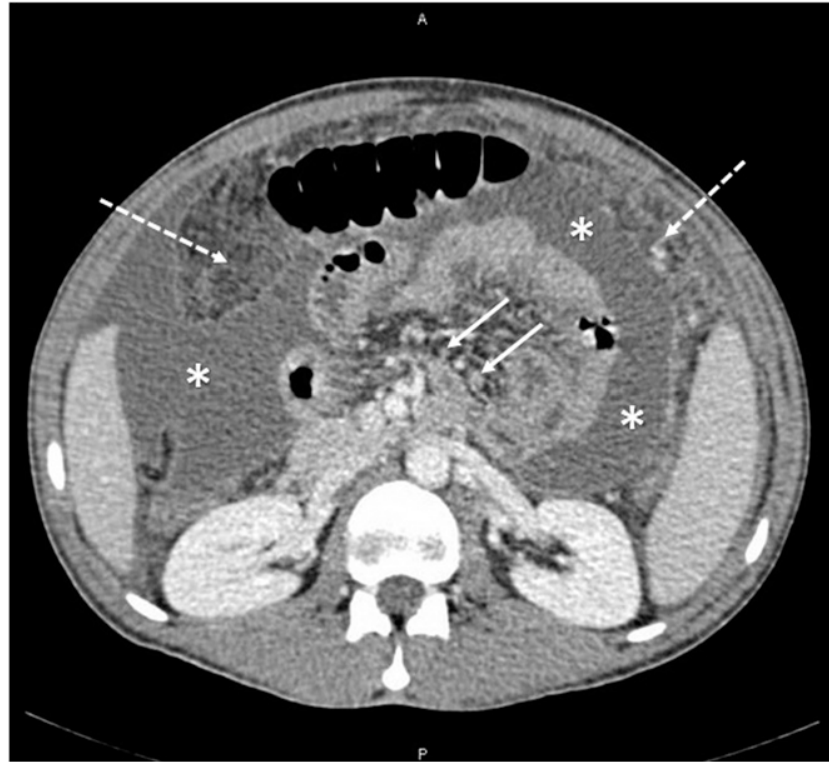

FIGURE 1. Computed tomographic scan of the abdomen with contrast depicting moderate to severe dense ascites (asterisks) with peritoneal thickening and omental caking (dashed arrows). Multiple subcentimetric lymph nodes (solid arrows) are seen at the root of the mesentery.

abdominal ultrasound showed no new changes. On day 36, after two negative consecutive COVID-19 PCR samples from the nasopharyngeal swab, the patient was discharged from the hospital to the quarantine facility and was scheduled for a peritoneal biopsy 2 weeks later.

On the day of the surgery, the patient looked tired and complained of a high temperature and shortness of breath. His

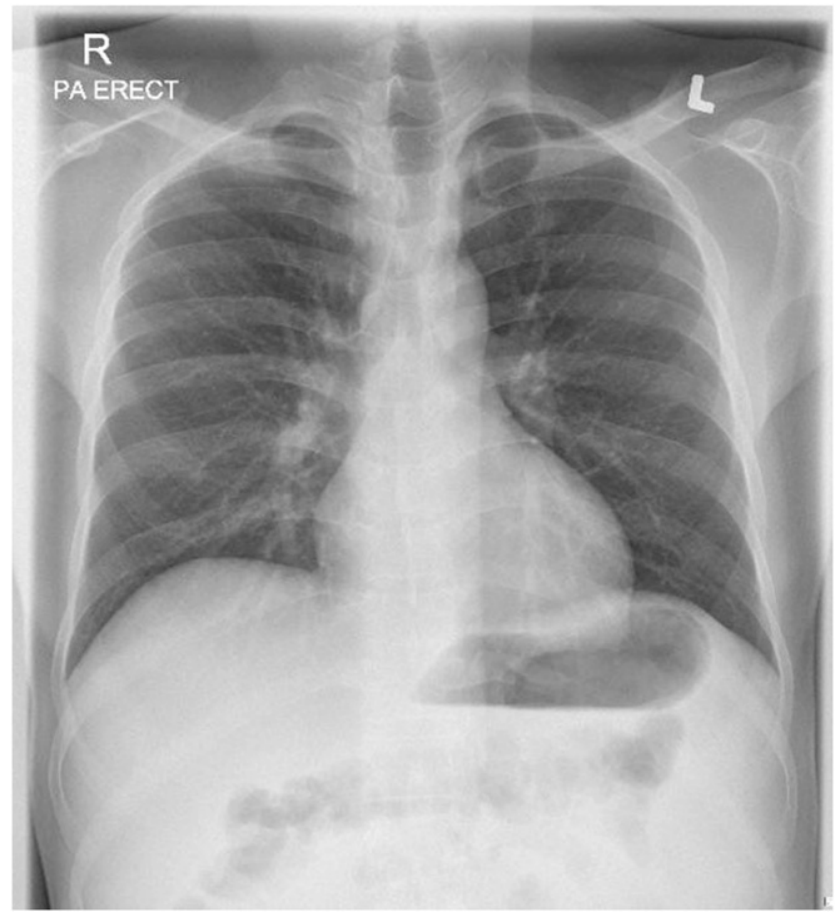

Figure 2. A normal chest X-ray at the first admission, before the diagnosis of COVID-19 infection.
TABLE 1

The analysis of peritoneal fluid, comparing glucose, lactate dehydrogenase, total protein, and albumin between peritoneal fluid and serum

\begin{tabular}{|c|c|c|}
\hline Variable & Peritoneal fluid & Serum \\
\hline Color & Turbid yellow & - \\
\hline White blood cells (cells $/ \mu \mathrm{L}$ ) & 500 & - \\
\hline Red blood cells (cells $/ \mu \mathrm{L})$ & 1,538 & - \\
\hline Neutrophils (\%) & 9 & - \\
\hline Lymphocyte (\%) & 65 & - \\
\hline Monocyte (\%) & 20 & - \\
\hline Macrophage (\%) & 6 & - \\
\hline Glucose (mmol/L) & 3.7 & 6.0 \\
\hline LDH (U/L) & 578 & 238 \\
\hline Protein (g/L) & 54.1 & 78 \\
\hline Albumin (g/L) & 22.3 & 25 \\
\hline SAAG (g/L) & & 2.7 \\
\hline
\end{tabular}

physical examination showed a temperature of $39^{\circ} \mathrm{C}$, a respiratory rate of 40-50 breaths per minute, and a heart rate of $120-130$ beats per minute. Oxygen saturation was $90 \%$ on a 10-L/min nonrebreather mask. So, the patient was transferred to the intensive care unit (ICU) and intubated. Chest X-ray showed bilateral, diffuse, coalescent miliary airspace opacities (Figure 3). Sputum and bronchoalveolar lavage (BAL) samples showed positive $M$. tuberculosis by PCR, and the drug susceptibility pattern did not show drug resistance. BAL showed scanty growth of Enterobacter cloacae. The respiratory viral panel, including the influenza virus from the BAL sample, was negative. Repeated SARS-CoV-2 RT-PCR from nasopharyngeal and throat swabs was negative.

The patient was started on antitubercular treatment (ethambutol 1,100 mg/d, isoniazid $300 \mathrm{mg} / \mathrm{d}$, rifampicin $600 \mathrm{mg} / \mathrm{d}$, and pyrazinamide $1,600 \mathrm{mg} / \mathrm{d}$ ). He completed intravenous amikacin for 10 days and piperacillin-tazobactam for 5 days. He improved dramatically on the antitubercular treatment and

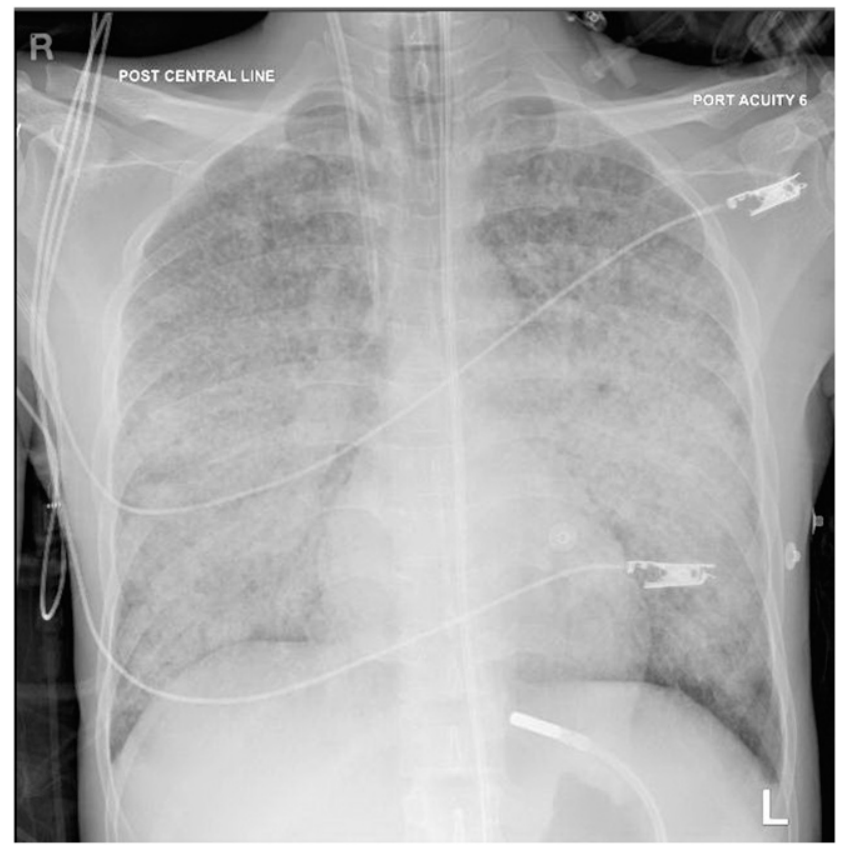

FlgURE 3. A chest X-ray at the second admission shows bilateral diffuse coalescent miliary airspace opacities. 
intravenous antibiotics, and was extubated 5 days after ICU admission. On day 9 of the second admission, the patient was transferred from the ICU to the standard medical ward. On day 18 , repeated abdominal ultrasound showed resolution of the ascitic fluid. On day 25, after two consecutive negative sputum AFB smears, the patient was discharged home with a follow-up appointment at the TB clinic.

\section{DISCUSSION}

COVID-19 Infection can be associated with other respiratory pathogens. ${ }^{10}$ We report a patient with possible peritoneal TB who progressed to miliary pulmonary TB after COVID-19 infection.

Few studies have reported the association between active TB and COVID-19 infection. ${ }^{11-16}$ Tadolini et al. ${ }^{12}$ reported 49 patients with concurrent infections, of whom 14 (28.5\%) were diagnosed with active TB infection more than 1 week after COVID-19 infection, and 9 (18.3\%) were diagnosed with COVID-19 and active TB simultaneously within the same week. Of the 49 patients, $13(26.5 \%)$ had extrapulmonary TB. In another cohort study, Stochino et al. ${ }^{13}$ identified 20 patients with concurrent COVID-19 and active TB. Nineteen patients had pulmonary TB, three of whom also had extrapulmonary involvement; one patient had the disseminated extrapulmonary form. Tham et al. ${ }^{15}$ reported four patients with TB and COVID-19 coinfections, where TB was diagnosed by clinical manifestation, radiological findings, and positive Interferon-Gamma Release Assay. The four patients showed improvement of symptoms after antitubercular treatment.

Few studies have reported a flare-up of TB after COVID-19 infection. Yao et al. ${ }^{14}$ reported two patients with a previous history of treated pulmonary TB who had pulmonary TB flareup after COVID-19 infection. One of them was a 50-year-old man who died of ARDS, although he was started on antitubercular treatment. Faqihi et al. ${ }^{16}$ reported a 60 -year-old patient who had a past history of TB. He developed COVID-19 infection and subsequently developed a pulmonary TB flare. Liu et al. ${ }^{11}$ reported a patient who was treated twice for TB previously and later was diagnosed with TB and COVID-19 coinfection. Stochino et al. ${ }^{13}$ reported that $35 \%$ (7 of 20 ) of patients with TB infection had chest worsening after COVID19 infection, as seen radiologically, which indicates that COVID-19 infection might lead to the worsening of TB infection.

The rapid deterioration of our patient resulting from the development of miliary pulmonary TB might have been accelerated by COVID-19 infection. Enterobacter cloacae growth from BAL could be a contamination from normal upper respiratory flora, as we did not have any evidence that the patient was immunocompromised. Still, we cannot exclude the possibility that it might have aggravated the patient's clinical course.

Host immune response is stimulated by active TB infection, and COVID-19 infection further increases the immune burden, which could lead to an out-of-control immune system and cytokine storm, inducing pulmonary and multiorgan dysfunction. ${ }^{11}$ TB reactivation can also be explained by T-cell exhaustion resulting from COVID-19 infection. A retrospective study observed that T-cell counts in COVID-19 patients were depleted significantly, and even the surviving T-cells were functionally exhausted. ${ }^{17}$ On the other hand, a recent report suggested that enhanced T-cell function is correlated with TB reactivation protection. ${ }^{18}$

Limitations. Hydroxychloroquine and azithromycin were used in the treatment of the mild COVID-19 infection in our patient according to the national protocol, which was based on earlier studies. ${ }^{19,20}$ The protocol has been updated after larger studies showed that these medications have no benefit. $^{21,22}$

The peritoneal biopsy was not done after COVID-19 infection resolution because the patient showed clinical improvement with complete resolution of the ascitic fluid after starting the antitubercular treatment.

Received September 7, 2020. Accepted for publication March 13, 2021.

Published online March 30, 2021.

Acknowledgment: Publication charges for this article were waived due to the ongoing pandemic of COVID-19.

Disclosure: We have read and complied with the policy of the journal on ethical consent, as stated in the guide to authors. The institutional review board has approved the work (manuscript no. MRC-04-20684).

Authors' addresses: Moustafa M. Elziny, Department of Family Medicine, Hamad Medical Corporation, Doha, Qatar, E-mail: drmostafaelzeiny@ gmail.com. Ahmed Ghazy, Department of Bariatric Medicine, Hamad Medical Corporation, Doha, Qatar, E-mail: dr.ahmedazmyghazy@ gmail.com. Khaled A. Elfert, Department of Gastroenterology, Hamad Medical Corporation, Doha, Qatar, E-mail: kh.elfert90@gmail.com. Mohamed Aboukamar, Department of Infectious Disease, Hamad Medical Corporation, Doha, Qatar, E-mail: mkamar@hamad.qa.

This is an open-access article distributed under the terms of the Creative Commons Attribution (CC-BY) License, which permits unrestricted use, distribution, and reproduction in any medium, provided the original author and source are credited.

\section{REFERENCES}

1. WHO, 2020. Tuberculosis. Available at: https://www.who.int/ news-room/fact-sheets/detail/tuberculosis. Accessed February $27,2021$.

2. Centers for Disease Control and Prevention, 2020. Tuberculosis. Global Health. Available at: https://www.cdc.gov/globalhealth/ newsroom/topics/tb/index.html. Accessed February 27, 2021.

3. Dheda K, Barry CE, Maartens G, 2016. Tuberculosis. Lancet 387: 1211-1226.

4. Piqueras AR, Marruecos L, Artigas A, Rodriguez C, 1987. Miliary tuberculosis and adult respiratory distress syndrome. Intensive Care Med 13: 175-182.

5. Mert $A$ et al., 2017. Miliary tuberculosis: epidemiological and clinical analysis of large-case series from moderate to low tuberculosis endemic Country. Medicine 96.

6. Al Kuwari $\mathrm{HM}$ et al., 2020. Epidemiological investigation of the first 5685 cases of SARS-CoV-2 infection in Qatar, 28 February-18 April 2020. BMJ Open 10: 40428.

7. Gandhi RT, Lynch JB, del Rio C, 2020. Mild or moderate COVID19. N Engl J Med, doi: 10.1056/nejmcp2009249.

8. Pirquet C, 1908. Das verhalten der kutanen tuberkulinreaktion während der masern. Dtsch Med Wochenschr 34: 1297-1300.

9. Moss AR, Hahn JA, Tulsky JP, Daley CL, Small PM, Hopewell PC, 2000. Tuberculosis in the homeless: a prospective study. Am J Crit Care Med 162. Available at: https://www.atsjournals.org/ doi/full/10.1164/ajrccm.162.2.9910055. Accessed February 5, 2021.

10. Kim D, Quinn J, Pinsky B, Shah NH, Brown I, 2020. Rates of coinfection between SARS-CoV-2 and other respiratory pathogens. JAMA 323: 2085-2086. 
11. Liu C et al., 2020. Severe COVID-19 cases with a history of active or latent tuberculosis. Int J Tuberc Lung Dis, doi: 10.5588/ ijtld.20.0163.

12. Tadolini $\mathrm{M}$ et al., 2020. Active tuberculosis, sequelae and COVID19 co-infection: first cohort of 49 cases. Eur Respir J 56, doi: 10.1183/13993003.01398-2020.

13. Stochino C, Villa S, Zucchi P, Parravicini P, Gori A, Raviglione MC, 2020. Clinical characteristics of COVID-19 and active tuberculosis co-infection in an Italian reference hospital. Eur Respir J 56: 2001708.

14. Yao $Z$ et al., 2020. Three patients with COVID-19 and pulmonary tuberculosis, Wuhan, China, January-February 2020. Emerg Infect Dis 26, doi: 10.3201/eid2611.201536.

15. Tham SM, Lim WY, Lee CK, Loh J, Premkumar A, Yan B, Kee A, Chai L, Tambyah PA, Yan G, 2020. Four patients with COVID-19 and tuberculosis, Singapore, April-May 2020. Emerg Infect Dis 26, doi: 10.3201/eid2611.202752.

16. Faqihi F, Alharthy A, Noor AF, Balshi A, Balhamar A, Karakitsos D, 2020. COVID-19 in a patient with active tuberculosis: a rare case-report. Respir Med Case Rep 31: 101146.
17. Diao B et al., 2020. Reduction and functional exhaustion of T cells in patients with coronavirus disease 2019 (COVID-19). medRxiv, doi: 10.1101/2020.02.18.20024364.

18. Foreman TW et al., 2016. CD4+ T-cell-independent mechanisms suppress reactivation of latent tuberculosis in a macaque model of HIV coinfection. Proc Natl Acad Sci USA 113: E5636-E5644.

19. Gautret $P$ et al., 2020. Hydroxychloroquine and azithromycin as a treatment of COVID-19: results of an open-label nonrandomized clinical trial. Int J Antimicrob Agents 56: 105949.

20. Chen $\mathrm{J}$ et al., 2020. A pilot study of hydroxychloroquine in treatment of patients with moderate COVID-19. Zhejiang Da Xue Xue Bao Yi Xue Ban 49: 215-219.

21. Furtado RHM et al., 2020. Azithromycin in addition to standard of care versus standard of care alone in the treatment of patients admitted to the hospital with severe COVID-19 in Brazil (COALITION II): a randomised clinical trial. Lancet 396: 959-967.

22. RECOVERY Collaborative Group, 2020. Effect of hydroxychloroquine in hospitalized patients with COVID-19. N Engl J Med 383: 2030-2040. 